\title{
Uso do delta check da creatinina como ferramenta para detecção precoce de lesão
}

\section{renal aguda: uma revisão de escopo}

\author{
Use of creatinine delta check as a tool for early detection of acute kidney injury: a scope review \\ Uso de creatinine delta check como herramienta para la detección temprana de lesión renal aguda:
} una revisión del alcance

Recebido: 26/11/2021 | Revisado: 03/12/2021 | Aceito: 17/12/2021 | Publicado: 02/01/2022

\author{
Gilvan Morais Neto Lira \\ ORCID: https://orcid.org/0000-0002-7489-7055 \\ Universidade Estadual do Ceará, Brasil \\ E-mail: Gilvan.lira@aluno.uece.br \\ Maria Salete Bessa Jorge \\ ORCID: https://orcid.org/0000-0001-6461-3015 \\ Universidade Estadual do Ceará, Brasil \\ E-mail: maria.salete.jorge@gmail.com \\ Cybelle Façanha Barreto Medeiros Linard \\ ORCID: https://orcid.org/0000-0001-7927-9320 \\ Universidade Estadual do Ceará, Brasil \\ Universidade Estácio do Ceará, Brasil \\ Centro Universitário Maurício de Nassau, Brasil \\ E-mail: cybellelinard@yahoo.com.br
}

\begin{abstract}
Resumo
A lesão renal aguda (LRA) é uma condição caracterizada pela redução da função renal. Existem vários métodos de diagnosticar esta condição, o mais aceito é o KDIGO, que classifica esta condição em 3 estágios. O delta check é a ferramenta laboratorial utilizada para calcular a variação entre as dosagens de um exame atual e seu anterior. O objetivo deste trabalho é verificar se o delta check pode ser utilizado como auxílio diagnóstico de LRA. Este estudo foi elaborado através de uma revisão de escopo da literatura existente com uso de palavras chave e descritores. As buscas nos bancos resultaram em 177 artigos onde após os processos de eliminação 6 artigos foram incluídos para o estudo. Os artigos tinham como origem os seguintes países: Reino Unido, Estados Unidos e Canadá, todos em língua inglesa. Cada artigo tinha regras diferentes para o cálculo do delta check e também diferentes limites de tempo entre as dosagens de creatinina evidenciando que cada instituição criou regras de acordo com a população atendida em suas unidades. Em todos os artigos o delta check teve o seu auxílio comprovado, mas no artigo de Kothari ganhou mais destaque devido além de criar um alerta com o delta check foi enfatizado a necessidade de treinamentos da equipe médica para o diagnóstico de LRA. Pode-se concluir que o delta check auxilia na LRA, sendo fundamental que cada instituição crie suas regras de delta check.
\end{abstract}

Palavras-chave: Creatinina; Delta Check; Laboratório; Laboratório clínico; Lesão renal aguda.

\begin{abstract}
Acute kidney injury (AKI) is a condintion characterized by reduced kidney function. There are several methods of diagnosing the condition, the most accepted is KDIGO, which classifies the disease into 3 stages. Delta check is the laboratory tool used to calculate the variation between the dosages of a current exam and your previous one. The objective of this work is to verify if the delta check can be used as a diagnostic aid for AKI. This study was developed through a scope review of the existing literature using keywords and descriptors. The searches in the databases resulted in 177 articles where after the elimination processes 6 articles were included for the study. The articles originated in the following countries: United Kingdom, United States and Canada, all in English. Each article had different rules for calculating the delta check and also different time limits between creatinine dosages, showing that each institution created rules according to the population served in their units. In all articles, delta check was proven to help, but in Kothari's article it gained more prominence because, in addition to creating an alert with delta check, the need for training of medical staff for the diagnosis of AKI was emphasized. It can be concluded that delta check helps in LRA, it is essential that each institution create its delta check rules.
\end{abstract}

Keywords: Acute kidney injury; Clinical laboratory; Creatinine; Delta Check; Laboratory.

\section{Resumen}

La lesión renal aguda (IRA) es una afección caracterizada por una función renal reducida. Existen varios métodos para diagnosticar esta condición, el más aceptado es KDIGO, que clasifica esta condición en 3 etapas. La verificación delta 
es la herramienta de laboratorio que se utiliza para calcular la variación entre las dosis de un examen actual y el anterior. El objetivo de este trabajo es verificar si la verificación delta se puede utilizar como ayuda para el diagnóstico de LRA. Este estudio se llevó a cabo mediante una revisión del alcance de la literatura existente utilizando palabras clave y descriptores. Las búsquedas en las bases de datos arrojaron 177 artículos donde luego de los procesos de eliminación se incluyeron 6 artículos para el estudio. Los artículos se originaron en los siguientes países: Reino Unido, Estados Unidos y Canadá, todos en inglés. Cada artículo tenía diferentes reglas para calcular el control delta y también diferentes límites de tiempo entre las dosis de creatinina, lo que demuestra que cada institución creaba reglas de acuerdo con la población atendida en sus unidades. En todos los artículos se demostró que el chequeo delta ayuda, pero en el artículo de Kothari ganó más protagonismo porque, además de crear una alerta con chequeo delta, se enfatizó la necesidad de capacitar al personal médico para el diagnóstico de LRA. Se puede concluir que la verificación delta ayuda en la LRA, siendo fundamental que cada institución cree sus reglas de verificación delta.

Palabras clave: Creatinina; Delta Check; Laboratorio; Laboratorio clínico; Lesión renal aguda.

\section{Introdução}

A Lesão Renal Aguda (LRA) é uma condição caracterizada pela redução abrupta da função renal evidenciada pela redução do débito urinário e aumento da concentração de creatinina sérica em um intervalo de curto espaço de tempo, ou seja, em algumas horas ou em poucos dias. A LRA é um problema que vem se tornando cada vez mais comum e sério em pacientes internados nos hospitais ao redor do mundo e está associada a uma consequência de condição clínica ou algum procedimento, tais como cirurgias ou processos infecciosos e também é associada a uma alta morbidade e mortalidade. Algumas das principais consequências da LRA tem sido a necessidade de o paciente passar por diálise, desenvolver doença renal crônica e até mesmo morte. Apesar dos avanços em tratamentos a incidência de LRA é alta, podendo acometer cerca de 0,3\% da população total, 18\% de pacientes internados e $60 \%$ de pacientes com doenças crônicas. Esses dados evidenciam a necessidade de um diagnóstico precoce de LRA para melhorar a saúde do paciente e assim reduzir os riscos que a doença traz (Baron et al., 2015; Flynn \& Dawnay, 2015; Kothari et al, 2018; Lima et al., 2020; Da Paixão Duarte et al., 2020; Siew \& Davenport, 2015; Silva et al., 2018; Thomas et al., 2015).

Existem 4 diretrizes classificatórias de LRA, o método RIFLE (Risk, Injury, Failure, Loss, End-Stage Renal Disease), o método AKIN (Acute Kidney Injury Network), o método de Waikar \& Bonventre e o mais atual o KDIGO (Kidney Disease: Improving Gloval Outcomes). O método RIFLE classifica a LRA em 5 fases, a primeira fase é chamado de RISCO e ocorre quando dentro de um período de 7 dias o valor de creatinina sérica aumenta em 1,5 vez maior quando comparado com o valor da creatinina basal do paciente e quando a Taxa de Filtração Glomerular ou TFG apresenta uma redução de $25 \%$ comparada com a basal, a segunda fase é definida como LESÃO e ocorre quando o valor da creatinina do paciente aumenta em 2 vezes quando comparada com o valor basal e quando a TFG apresenta uma redução de 50\%, a terceira fase é chamada de Falha e ocorre quando a creatinina do paciente apresenta um valor 3 vezes maior que o valor basal e com TFG reduzida em $75 \%$ ou quando o paciente apresenta um valor de creatinina maior ou igual a $4 \mathrm{md} / \mathrm{dL}$, a quarta fase é chada de Perda da Função que ocorre quando os valores do estágio três se mantém por mais de 4 semanas e o última fase chamado Terminal que ocorre quando os valores do terceiro estágio se mantém por mais de 3 meses (Bellomo et al., 2004; Maria et al., 2021; Mehta et al., 2007; Silva et al., 2018; Waikar \& Bonventre, 2009)..

A metodologia AKIN divide a classificação de LRA em três estágios, o primeiro estágio ocorre quando o paciente apresenta um aumento no valor de creatinina sérica igual ou maior que $0,3 \mathrm{mg} / \mathrm{dL}$ ou quando apresenta um valor de 1,5 até 2 vezes maior que o valor basal de creatinina do paciente ou quando a depuração de urina é menor que $0,5 \mathrm{ml} / \mathrm{Kg}$ por hora por mais de 6 horas, o segundo estágio é determinado quando o paciente apresenta um valor de creatinina maior que 2 vezes e menor que 3 vezes do valor basal ou quando apresenta uma depuração de urina menor que $0,5 \mathrm{ml} / \mathrm{Kg}$ por hora por mais de 12 horas e o terceiro estágio ocorre quando o paciente apresenta um valor de creatinina maior que 3 vezes o valor basal ou quando apresenta um valor de creatinina maior que $4 \mathrm{mg} / \mathrm{dL}$. Já o método proposto por Waikar e Bonventre também classifica a LRA em três 
estágios, sendo que o primeiro estágio ocorre quando há um aumento no valor da creatinina sérica do paciente maior ou igual a $0,3 \mathrm{mg} / \mathrm{dL}$ em um período de até 24 horas ou um aumento maior ou igual a $0,5 \mathrm{mg} / \mathrm{dL}$ em um período de até 48 horas, o segundo estágio ocorre quando o paciente apresenta um aumento no valor de creatinina maior ou igual a $0,5 \mathrm{mg} / \mathrm{dL}$ em um período de até 24 horas ou quando apresenta um aumento maior ou igual a 1,0mg/dL em um período de até 48 horas e o terceiro estágio ocorre quando o paciente apresenta um aumento no valor da creatinina maior ou igual a $1,0 \mathrm{mg} / \mathrm{dL}$ em um período de até 24 horas ou um aumento maior ou igual a 1,5mg/dL em um período de até 48 horas (Bellomo et al., 2004; Maria et al., 2021; Mehta et al., 2007; Silva et al., 2018; Waikar \& Bonventre, 2009).

O método KDIGO que atualmente é o mais utilizado para o diagnóstico de LRA classifica a condição em 3 estágios, onde o estágio 1 ocorre quando o paciente apresenta um valor de creatinina de 1,5 até 1,9 vezes o valor basal ou quando apresenta um aumento de $0,3 \mathrm{mg} / \mathrm{dL}$ no valor de creatinina em um período de até 48 horas ou quando apresenta uma depuração urinária menor que $0,5 \mathrm{ml} / \mathrm{Kg} / \mathrm{h}$ por um período de 6 a 12 horas, o 2 estágio ocorre quando o paciente apresenta um valor de creatinina de 2 a 2,9 vezes o valor da creatinina basal ou quando apresenta uma depuração urinária menor que $0,5 \mathrm{mg} / \mathrm{Kg} / \mathrm{h}$ por um período maior ou igual a 12 horas e o 3 estágio ocorre quando o paciente apresenta um valor de creatinina maior ou igual a 3 vezes o valor da creatinina basal ou quando apresenta um valor de creatinina maior ou igual a $4 \mathrm{mg} / \mathrm{dL}$ ou quando é iniciada a terapia de substituição renal ou quando o paciente tem idade menor que 18 anos e apresenta uma TFG menor que $35 \mathrm{ml} / \mathrm{min}$ per $1,73 \mathrm{~m}^{2}$ ou quando apresenta uma depuração urinária menor que $0,3 \mathrm{ml} / \mathrm{Kg} / \mathrm{h}$ por um período maior ou igual a 24 horas ou quando apresenta anuria, ausência de urina, por um período igual ou maior que 12 horas(Bellomo et al., 2004; Maria et al., 2021; Mehta et al., 2007; Silva et al., 2018; Waikar \& Bonventre, 2009).

O delta-check é uma ferramenta que no uso laboratorial consiste na análise da diferença entre o valor atual de um determinado exame com o valor anterior desde mesmo exame do paciente. Inicialmente essa ferramenta foi utilizada para detectar possíveis erros ocorridos durante a coleta de sangue dos pacientes, entretanto com o desenvolvimento de novas tecnologias, como a introdução da identificação por códigos de barras, a segurança na coleta de sangue dos pacientes aumentou reduzindo a eficácia do delta check em detectar erros de coleta. Atualmente o delta check tem sido utilizado para monitorar as variações dos resultados dos exames e assim auxiliar no diagnóstico clínico dos pacientes(Gruenberg et al., 2018; Randell \& Yenice, 2019; Straseski \& Strathmann, 2013).

Por se tratar de uma ferramenta que já é usualmente utilizada em diversos laboratórios clínicos este trabalho tem como objetivo verificar através de uma revisão na literatura como o uso do delta check pode ser útil na detecção precoce de LRA auxiliando os médicos no correto diagnóstico por um baixo custo.

\section{Método}

Este estudo foi elaborado como uma revisão de escopo. A revisão de escopo é um método que busca sintetizar as evidências de pesquisa e mapear a literatura existente de um determinado assunto. A pergunta norteadora utilizada para realizar este artigo foi criada com base na estratégica PCC, que é uma sigla para ajudar a identificar os pontos chaves que são Problema, Conceito e Contexto, sendo o P - pacientes com Lesão Renal Aguda, C - uso do delta check da creatinina e C - diagnóstico, sendo ela construída da seguinte forma: “O delta check da creatinina pode ser útil para diagnosticar Lesão Renal Aguda?”. Foram incluídos estudos que retratassem como o delta check poderia ajudar no diagnóstico precoce de Lesão Renal Aguda que tenham sido publicados em inglês ou português. Para a busca dos artigos foram utilizados os seguintes descritores decs/mesh: Acute Kidney Injury e Creatinine; e as seguintes palavras chaves: delta check, clinical laboratory e laboratory; formulando a seguinte equação de busca "(delta check) and ((clinical laboratory) or (laboratory)) and (acute kidney injury) and (creatinine)". A busca de dados foi realizada nos seguintes bancos: Biblioteca Virtual em Saúde (BVS), Portal de Periódicos CAPES/MEC, National 
Library of Medicine (PUBMED) e EMBASE. A busca nos bancos foi realizada nos dias 01 a 05 de Julho de 2021 (Aromataris \& Munn 2020).

A escolha dos artigos foi dividida em quatro etapas. A $1^{\text {a }}$ etapa foi a realização da busca dos artigos nas bases escolhidas e a eliminação dos artigos repetidos. $\mathrm{Na} 2^{\mathrm{a}}$ etapa foram analisados os títulos e resumos dos artigos restantes após a $1^{\mathrm{a}}$ etapa, como critério de exclusão foram adotados artigos que estivessem duplicados ou repetidos nos bancos, que não fossem relevantes ao objetivo da pesquisa, que não estivessem disponíveis de forma completa ou que não estivessem nos idiomas inglês, português ou espanhol. $\mathrm{Na} 3^{\mathrm{a}}$ etapa foi realizada a análise integral dos textos dos artigos que não foram eliminados na etapa anterior e então a $4^{\text {a }}$ etapa onde após revisão integral dos textos aqueles que eram pertinentes ao estudo foram selecionados (Aromataris \& Munn 2020).

\section{Resultados}

A equação de busca utilizada nos bancos resultou em 177 artigos, sendo que 9 foram removidos por estarem duplicados. Após leitura e análise dos resumos 162 artigos foram excluídos. Totalizando em 5 artigos para leitura integral e todos foram elegíveis para este trabalho. Figura 1 mostra de forma clara todas as etapas seguidas. Todos os artigos selecionados para compor o trabalho estavam em língua inglesa.

Figura 1. Fluxograma da seleção de artigos.

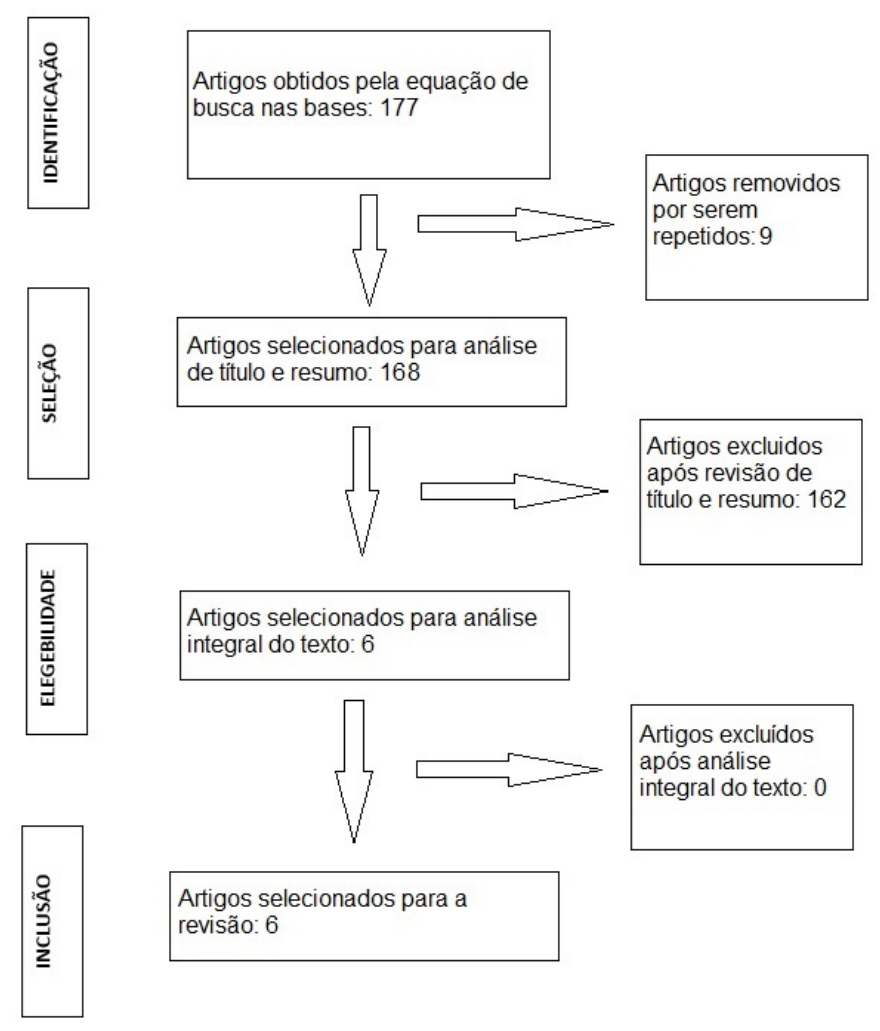

Fonte: Autores.

As 6 publicações incluídas para análise integral do texto foram publicadas entre os anos de 2012 e 2018 , onde 3 foram publicadas em revistas do Reino Unido, 2 em revistas americanas e 1 em uma revista no Canadá. O Quadro 1 apresenta de forma sintética as conclusões obtidas após análise dos artigos incluídos na análise e o Quadro 2 mostra os tipos de estudos realizados de acordo com o país onde o artigo foi publicado. 
Quadro 1. Analise dos artigos.

\begin{tabular}{|l|l|l|}
\hline Autor(es) / Ano & Título & Revista de Publicação \\
\hline Garner et al, 2012 & $\begin{array}{l}\text { Detection of patients with acute kidney } \\
\text { injury by the clinical laboratory using } \\
\text { rises in serum creatinine: comparison } \\
\text { of proposed definitions and a } \\
\text { laboratory delta check }\end{array}$ & $\begin{array}{l}\text { Annals of Clinical } \\
\text { Biochemistry }\end{array}$ \\
\hline Thomas et al, 2015 & $\begin{array}{l}\text { Earlier intervention for acute kidney } \\
\text { injury: evaluation of an outreach } \\
\text { service and a long-term follow-up }\end{array}$ & $\begin{array}{l}\text { Nephrology } \\
\text { Transplantation }\end{array}$ \\
\hline Baron et al, 2015 & $\begin{array}{l}\text { Enhanced Creatinine and Estimated } \\
\text { Glomerular Filtration Rate Reporting } \\
\text { to Facilitate Detection of Acute } \\
\text { Kidney Injury }\end{array}$ & $\begin{array}{l}\text { American Society for } \\
\text { Clinical Pathology }\end{array}$ \\
\hline Flynn \& Dawny, 2015. & $\begin{array}{l}\text { A simple electronic alert for acute } \\
\text { kidney injury }\end{array}$ & $\begin{array}{l}\text { The Association of Clinical } \\
\text { Biochemistry \& Laboratory } \\
\text { Medicine }\end{array}$ \\
\hline Kothari et al, 2018 & $\begin{array}{l}\text { Impact of Daily Electronic Laboratory } \\
\text { Alerting on Early Detection and } \\
\text { Clinical Documentation of Acute } \\
\text { Kidney Injury in Hospital Settings }\end{array}$ & Academic Pathology \\
\hline Randell \& Yenice, & $\begin{array}{l}\text { Delta Checks in the clinical laboratory } \\
2019\end{array}$ & $\begin{array}{l}\text { Critical Reviews in Clinical } \\
\text { Laboratory Sciences }\end{array}$ \\
\hline
\end{tabular}

\section{Objetivo do estudo}

Comparar os métodos de diagnóstico de LRA (RIFLE, AKIN e Waikar \& Bonventre) através de seus critérios de

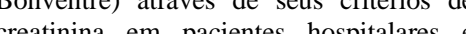
avaliar a peforme avaliar a performa check para delta para identificação de pacientes com LRA.

Avaliação do impacto da análise de creatinina pelos limites de referência e desenvolver e implementar um algoritmo melhorado de creatinina

Descrever como um simples alerta eletrônico automático de delta check pode ser implantado pela equipe do laboratório com mínimos custos e não requerer operação manual de um bioquímico.

Investigar os efeitos de alerta diário aboratorial de pacientes com risco de LRA de acordo com diagnósticos documentados pela Classificação Internacional de

Obję̧ivo de

Detivo devisão é prover ao leitor deste artigo uma compreensão geral sobre delta checks, especificamente para o seu uso para detecção de erros e promover o seu uso racional.
Avaliação do uso de um alerta eletrônico
Conclusão após análise

Como resultados de suas análises durante o Mês de Outubro de 2008 mostrou que o método RIFLE diagnosticou um total de 94 pacientes, o método AKIN diagnosticou um total de 125 pacientes, o método Waikar \& Bonventre diagnosticou um total de 100 pacientes, e utilizando o delta check foram diagnosticados um total 146 pacientes sendo que o total de pacientes diagnosticados com LRA foi de 149 pacientes. O delta check foi capaz de detectar $98 \%$ de todos os casos de LRA, evidenciando sua real utilidade no auxilio diagnóstico da doença.

Foi realizada a criação de um alerta eletrônico baseado na variação de $75 \%$ do delta check da creatinina. Foi feita uma comparação de período antes e depois da implantação do alerta eletrônico e houve um aumento na deteç̧ão de casos de LRA. Também é relatado no artigo que houve um aumento na sobrevivência a LRA imediato devido a uma interferência no paciente pela detecção precoce de LRA.

Realizaram estudo no Hospital Geral de Massachusetts, onde eles adotaram o critério do delta check como uma das formas de identificar pacientes com LRA. Eles obtiveram resultados absoluto de creatinina, entretanto quando o delta check foi combinado com os alertas e a regra de rastreamento mínima de creatinina os resultados foram ainda melhores, apresentando uma especificidade de $95 \%$ e uma sensibilidade de $94 \%$.

Foi descrito a implantação de um alerta eletrônico de delta check para a detecção de LRA pelo método AKIN. Após a criação do alerta, durante um período de 12 dias 88 pacientes receberam o alerta de possível LRA, destes 61(69,3\%) foram diagnosticados com LRA. Também é evidenciado que o alerta eletrônico de delta check pode ajudar a identificar LRA a qualquer momento com o mínimo de custo para o laboratório.

Além do alerta eletrônico de delta check de creatinina foi também realizado um treinamento de médicos(as) e enfermeiros(as), como resultado da combinação dos dois os diagnósticos de LRA durante o período de análise em 2014 de 4,5\% subiu para 8,32\% em 2015, 11,02\% em 2016 e $10,77 \%$ em 2017.

Neste estudo foi relatado como o delta check da creatinina pode representar um importante fator para o diagnóstico de LRA. Foram citados alguns estudos sobre o uso do delta check e embora cada estudo tenha utilizados diferentes critérios para o delta check em todos o delta check foi caracterizado com uma importante ferramenta para auxiliar o diagnóstico de LRA.

Fonte: Autores 
Quadro 2. Tipos de estudo.

\begin{tabular}{|l|l|l|}
\hline Título & Tipo de Estudo & Ano de Publicação \\
\hline $\begin{array}{l}\text { Detection of patients with acute kidney injury by the clinical } \\
\text { laboratory using rises in serum creatinine: comparison of } \\
\text { proposed definitions and a laboratory delta check }\end{array}$ & Estudo qualitativo retrospectivo & 2012 \\
\hline $\begin{array}{l}\text { Earlier intervention for acute kidney injury: evaluation of an } \\
\text { outreach service and a long-term follow-up }\end{array}$ & Estudo prospectivo. & 2014 \\
\hline $\begin{array}{l}\text { Enhanced Creatinine and Estimated Glomerular Filtration } \\
\text { Rate Reporting to Facilitate Detection of Acute Kidney } \\
\text { Injury }\end{array}$ & Estudo experimental retrospectivo & 2015 \\
\hline A simple electronic alert for acute kidney injury & Estudo qualitativo retrospectivo & 2015 \\
\hline $\begin{array}{l}\text { Impact of Daily Electronic Laboratory Alerting on Early } \\
\text { Detection and Clinical Documentation of Acute Kidney } \\
\text { Injury in Hospital Settings }\end{array}$ & Estudo quasi-experimental prospectivo. & 2018 \\
\hline Delta Checks in the clinical laboratory & Revisão de escopo & 2019 \\
\hline
\end{tabular}

Fonte: Autores.

\section{Discussão}

É possível observar que todos os artigos utilizam o delta check para ajudar a identificar precocemente casos de LRA, entretanto todos eles mudam a forma como usam o delta check, um exemplo disso temos no artigo de Garner et al. (2012), no qual o seu estudo utiliza o delta check como cálculo de diferença absoluta e o alerta era emitido quando valor do delta check era superior a $26 \mu \mathrm{mol} / \mathrm{L}$, já no artigo de Kothari et al, 2018 o valor de alerta do delta check era gerado quando valor era superior a 0,3 mg/dL, no estudo de Flynn e Dawnay (2015), o alerta era emitido quando o delta check apresentava valor maior que $50 \mu \mathrm{mol} / \mathrm{L}$, já o de Thomas et al (2015) determinou que o alerta era emitido quando o delta check de creatinina fosse superior ou igual a 75\%. Em cada estudo o delta check foi adaptado para a realidade dos pacientes atendidos, evidenciado a importância da análise da população atendida pela instituição para melhor uso das ferramentas de auxílio diagnóstico (Garner et al., 2012; Kothari et al., 2018).

Um outro ponto analisado fica entre os artigos experimentais foi avaliação de tempo entre as dosagens de creatinina utilizada para realizar a análise de delta check, onde como mostra o quadro 3 os tempos de análises foram diferentes em cada instituição. Muito embora todas as decisões tenham sido tomadas de acordo com equipe médicas um período muito longo entre dosagens de creatinina pode levar a um número alto de alertas falsos uma vez que outros fatores poderão influenciar na elevação da creatinina e que podem não estar diretamente ligados a LRA.

Quadro 3. Relação do tempo entre dosagens de creatinina para cálculo do delta check.

\begin{tabular}{|l|l|l|}
\hline Autor(es) / Ano & Tempo entre dosagens & Limite do Delta Check \\
\hline Garner et al, 2012 & 30 dias & $>26 \mu \mathrm{mol} / \mathrm{L}$ \\
\hline Thomas et al, 2015 & Não estabelecido & $>$ ou=75\% \\
\hline Baron et al, 2015 & 72 horas & $>$ ou igual a 1,5 vezes o valor da creatinina basal \\
\hline Flynn \& Dawny, 2015. & 90 dias & $\begin{array}{l}\text { Aumento de 50\% desde que o valor da } \\
\text { creatinina seja superior a 50 } \mu \text { mol/L }\end{array}$ \\
\hline Kothari et al, 2018 & $\begin{array}{l}>0,3 \mathrm{mg} / \mathrm{dL} \text { entre a creatinina atual com a } \\
\text { creatinina basal em ate 48 horas }\end{array}$ \\
\hline
\end{tabular}

Fonte: Autores.

$\mathrm{O}$ artigo de Kothari et al mostra um impacto maior do que os outros estudos, devido além do alerta eletrônico de delta check também ter sido realizado um treinamento dos médicos(as) e enfermeiros(as) acerca do diagnóstico de LRA, e o resultado da implantação das duas medidas foi um aumento substancial dos casos de LRA mesmo com o laboratório tendo pouco aumento de casos identificados, evidenciando que muitos pacientes desenvolviam LRA e não eram corretamente diagnosticados. O número absoluto de casos de LRA de janeiro a junho de 2014, período de análise antes da implantação do alerta e do treinamento, 
foi de 2965 casos, durante o mesmo período em 2015 após as medidas realizadas o número de casos saltou para 5523 casos, 7589 casos no mesmo período em 2016 e para 7383 casos em 2017. Um dado adicional que pode ser visto no artigo é que o diagnóstico de Necrose Tubular Aguda condição similar à LRA onde o número de casos durante o período de análise em 2014 foi de 789, em 2015 foi de 1040 casos, em 2016 foi de 1198 e em 2017 foram 1173.

De tal modo, os resultados da revisão poderão contribuir no projeto de tratamento do paciente, com a detecção precoce de lesão renal. Com isso, a identificação precoce de lesão renal favorece o diagnóstico de lesão renal para evitar desfechos adversos, como a lesão renal crônica ou a perca da função renal.

\section{Conclusão}

Após análise de todos os artigos o uso de um alerta eletrônico de delta check além de ser uma ferramenta extremamente útil também requer baixo custo para o laboratório para ser implementada. Entretanto é possível ver que em todos os artigos existem vários alertas que são considerados falso-positivos e uma forma de amenizar o trabalho é proposta no artigo de Kothari, através de um relatório diário de todos os pacientes que tiveram alertas de delta check para a 1 ronda hospitalar do dia. Podemos concluir que o uso do delta check para o auxílio diagnóstico de LRA é muito útil, mas que também é necessário que toda a equipe médica esteja treinada para realizar o correto diagnóstico de LRA.

\section{Referências}

Aromataris E, Z M. (2020). Jbimanual For Evidence Synthesis. E A, Z M, editors. https://synthesismanual.jbi.global. https://doi.org/10.46658/JBIMES-20-01

Baron, J. M., Cheng, X. S., Bazari, H., Bhan, I., Lofgren, C., Jaromin, R. T., Lewandrowski, K. B., et al. (2015). Enhanced creatinine and estimated glomerular filtration rate reporting to facilitate detection of acute kidney injury. American Journal of Clinical Pathology, 143(1), 42-49.

Bellomo, R., Ronco, C., Kellum, J. A., Mehta, R. L., \& Palevsky, P. (2004). Acute renal failure - definition, outcome measures, animal models, fluid therapy and information technology needs: the Second International Consensus Conference of the Acute Dialysis Quality Initiative (ADQI) Group. Critical care (London, England), 8(4).

Flynn, N., \& Dawnay, A. (2015). A simple electronic alert for acute kidney injury. Annals of Clinical Biochemistry, 52(2), 206-212.

Garner, A. E., Lewington, A. J. P., \& Barth, J. H. (2012). Detection of patients with acute kidney injury by the clinical laboratory using rises in serum creatinine: Comparison of proposed definitions and a laboratory delta check. Annals of Clinical Biochemistry, 49(1), 59-62.

Gruenberg, J. M., Stein, T. A., \& Karger, A. B. (2018). Determining the utility of creatinine delta checks: A large retrospective analysis. Clinical Biochemistry, 53(December 2017), 139-142. Elsevier. Retrieved from https://doi.org/10.1016/j.clinbiochem.2018.01.023

Kothari, T., Jensen, K., Mallon, D., Brogan, G., \& Crawford, J. (2018). Impact of Daily Electronic Laboratory Alerting on Early Detection and Clinical Documentation of Acute Kidney Injury in Hospital Settings. Academic Pathology, 5, 237428951881650.

Lima, W. L. de, Paula, L. B. de, Duarte, T. T. da P., \& Magro, M. C. da S. (2020). Conhecimento dos enfermeiros da atenção primária à saúde sobre fatores de risco para Lesão Renal Aguda. Escola Anna Nery, 24(2), 1-7.

Maria, J., Silva, M., Mauricio, C., Castro, C. De, Pismel, C., Castilho, M., Melo, E. R., et al. (2021). Manejo da lesão renal aguda: uma revisão narrativa Management of acute kidney injury : a narrative review Tratamiento de la lesión renal aguda : una revisión narrativa. Revista Eletrônica Acervo Saúde, 13(5), $1-8$.

Mehta, R. L., Kellum, J. A., Shah, S. V., Molitoris, B. A., Ronco, C., Warnock, D. G., Levin, A., et al. (2007). Acute kidney injury network: Report of an initiative to improve outcomes in acute kidney injury. Critical Care, 11(2), 1-8.

Da Paixão Duarte, T. T., Chagas Costa, L., De Lima, W. L., \& Da Silva Magro, M. C. (2020). Influência De Fatores Clínicos Na Lesão Renal Aguda Tt Influencia De Factores Clínicos En La Lesión Renal Aguda Tt - Influence of Clinical Factors on Acute Kidney Injury. Ciencia y enfermería, 26. http://www.scielo.cl/scielo.php?script=sci_arttext\&pid=S0717-95532020000100205\&lang=pt\%0Ahttp://www.scielo.cl/pdf/cienf/v26/0717-9553-cienf-26-

6.pdf

Randell, E. W., \& Yenice, S. (2019). Delta Checks in the clinical laboratory. Critical Reviews in Clinical Laboratory Sciences, 56(2), 75-97. Taylor \& Francis. Retrieved from https://doi.org/10.1080/10408363.2018.1540536

Siew, E. D., \& Davenport, A. (2015). The growth of acute kidney injury: A rising tide or just closer attention to detail? Kidney International, 87(1), 46-61. Elsevier Masson SAS. Retrieved from http://dx.doi.org/10.1038/ki.2014.293

Silva, H. M. da, Duarte, T. T. da P., \& Magro, M. C. da S. (2018). Influence of variation of the serum creatinine on outcomes of patient with acute kidney injury. Revista da Rede de Enfermagem do Nordeste, 19, e33348. 
Research, Society and Development, v. 11, n. 1, e6011123954, 2022

(CC BY 4.0) | ISSN 2525-3409 | DOI: http://dx.doi.org/10.33448/rsd-v11i1.23954

Straseski, J. A., \& Strathmann, F. G. (2013). Patient Data Algorithms. Clinics in Laboratory Medicine, 33(1), 147-160. Elsevier Inc. http://dx.doi.org/10.1016/j.cll.2012.11.009

Thomas, M. E., Sitch, A., Baharani, J., \& Dowswell, G. (2015). Earlier intervention for acute kidney injury: Evaluation of an outreach service and a long-term follow-up. Nephrology Dialysis Transplantation, 30(2), 239-244.

Waikar, S. S., \& Bonventre, J. V. (2009). Creatinine kinetics and the definition of acute kidney injury. Journal of the American Society of Nephrology, 20(3), 672-679. 